\section{Prevalência e fatores associados à obesidade em idosos residentes em Pelotas, Rio Grande do Sul, Brasil: classificação da obesidade segundo dois pontos de corte do índice de massa corporal}

\author{
Obesity prevalence and associated factors in the \\ elderly in Pelotas, Rio Grande do Sul State, \\ Brazil: obesity classification according to two \\ cutoff points for body mass index
}

\author{
${ }^{1}$ Faculdade de Nutrição, \\ Universidade Federal de \\ Goiás, Goiânia, Brasil. \\ 2 Núcleo de Estudos em \\ Saúde Coletiva, Universidade \\ Federal de Goiás, Goiânia, \\ Brasil. \\ 3 Instituto de Nutrição Josué \\ de Castro, Universidade \\ Federal do Rio de Janeiro, \\ Rio de Janeiro, Brasil. \\ 4 Secretaria de Estado da \\ Saúde, Goiânia, Brasil. \\ Correspondência \\ E. A. Silveira \\ Faculdade de Nutrição, \\ Universidade Federal de \\ Goiás. \\ Rua 227, Quadra $68 \mathrm{~s} / \mathrm{n}^{\circ}$, \\ Setor Leste Universitário, \\ Goiânia, GO \\ 74605-080, Brasil. \\ erikasil@terra.com.br
}

\begin{abstract}
The aim of this study was to estimate obesity prevalence and associated factors in the elderly, according to two cutoff points for body mass index (BMI). This was a cross-sectional, population-based study of a sample of 596 elderly residents in Pelotas, southern Brazil. Nutritional status was evaluated through BMI based on self-reported weight and stature. Poisson multiple regression with hierarchical analysis was applied, with two dependent variables for definition of obesity: $B M I>$ $27 \mathrm{~kg} / \mathrm{m}^{2}$ and $B M I \geq 30 \mathrm{~kg} / \mathrm{m}^{2}$, the cutoff points proposed by Lipschitz and the World Health Organization, respectively. Prevalence of obesity was 48.7\% (95\%CI: 44.6-52.7) for BMI > $27 \mathrm{~kg} / \mathrm{m} 2$ and $25.3 \%$ (95\% CI: 21.9-29.0) for BMI $\geq 30 \mathrm{~kg} / \mathrm{m}^{2}$. Two multiple regression models showed an association between obesity and female gender and age. BMI $>27 \mathrm{~kg} / \mathrm{m}^{2}$ showed an association with age and smoking and BMI $\geq 30 \mathrm{~kg} / \mathrm{m}^{2}$ with sedentary lifestyle. Obesity prevalence was high in this group of elderly. For the Brazilian elderly population and from a public health perspective, the study suggests the use of a more sensitive cutoff point, namely $B M I>27 \mathrm{~kg} / \mathrm{m}^{2}$.
\end{abstract}

Aged; Obesity; Body Mass Index; Nutritional Assessment; Prevalence
Erika Aparecida Silveira 1,2

Gilberto Kac ${ }^{3}$

Larissa Silva Barbosa 1,4

\section{Introdução}

O recente aumento da população idosa (60 anos e mais), em decorrência de modificações demográficas como a esperança de vida, vem se tornando uma das principais preocupações no campo da saúde, exigindo pesquisas e políticas públicas adequadas a essa nova realidade. Como conseqüência do envelhecimento populacional, verificam-se mudanças no perfil epidemiológico e nutricional, com predomínio das doenças crônicas não transmissíveis, próprias das faixas etárias mais avançadas 1 .

Dados da Pesquisa de Orçamentos Familiares (POF), realizada em 2002-2003, evidenciaram que a prevalência de obesidade, avaliada pelo índice de massa corporal (IMC) igual ou superior a $30 \mathrm{~kg} / \mathrm{m}^{2}$, na população brasileira, aumentou com a idade e atingiu $17,1 \%$ na faixa etária de 55 a 64 anos, $14 \%$ na categoria de 65 a 74 anos e 10,5\% nos idosos com 75 anos e mais 2 .

Os estudos epidemiológicos sobre o estado nutricional em idosos indicam que os distúrbios nutricionais estão associados com o risco de morbidade e de mortalidade ${ }^{3,4}$. Apesar de ser um preditor importante para morbidade e mortalidade na população idosa, pouco se sabe sobre a relação entre IMC e envelhecimento 4,5. Uma lacuna, em particular, diz respeito à ausência de consenso sobre o ponto de corte de IMC mais adequado para classificação antropométrica da obesidade. Entre os critérios mais utilizados para 
essa avaliação, estão o recomendado pela Organização Mundial da Saúde (OMS) ${ }^{4}$ e o proposto por Lipschitz et al. ${ }^{6}$.

O critério recomendado pela OMS 4 foi utilizado em inquéritos nacionais como a Pesquisa Nacional sobre Saúde e Nutrição (PNSN) 7 e a POF $2002 / 20032$ 2. No entanto, há críticas sobre o uso dos mesmos pontos de corte para classificar obesidade em adultos e idosos, pois mudanças na composição corporal, associadas ao processo de envelhecimento, devem ser consideradas 4,8 .

Já o critério de classificação proposto por Lipschitz et al. 6 assemelha-se ao utilizado pelo Centers of Disease Control and Prevention (CDC) no National Health and Nutrition Examination Survey III (NHANES III), realizado entre 1988 e $1994{ }^{9}$. Esse critério considera diferenças entre os sexos, porém não faz distinção para categorias com diferentes níveis de risco, ou seja, baixo peso, sobrepeso e obesidade ${ }^{9}$. Essa classificação leva em consideração as mudanças na composição corporal que ocorrem com o envelhecimento, uma vez que os idosos apresentam decréscimo da estatura, diminuição da quantidade de água e massa magra no organismo e maior porcentagem de gordura, quando comparados com os indivíduos adultos ${ }^{8}$. Nutrition Screening Initiative (NSI) 10 adotou o mesmo ponto de corte de Lipschitz et al. 6 para excesso de peso, porém com valor superior para a classificação de baixo peso. Assim, não há ainda um consenso sobre o valor ou ponto de corte de IMC mais adequado para avaliar obesidade em idosos, inclusive, não há, no Brasil, estudo de base populacional que permita avaliar a adequação do IMC como marcador de adiposidade em idosos 5 .

A presente investigação tem como objetivo estimar a prevalência e os fatores associados à obesidade em idosos, utilizando, como desfecho, a obesidade avaliada segundo dois pontos de corte de IMC. O artigo busca ainda discutir e comparar os resultados segundo os diferentes pontos de corte empregados.

\section{Métodos}

Realizou-se estudo de delineamento transversal, de base populacional, em uma amostra probabilística de adultos, residentes na zona urbana do Município de Pelotas, Rio Grande do Sul, Brasil, entre novembro de 1999 e janeiro de 2000.

Dos 259 setores censitários do município, foram sorteados sistematicamente $48 \mathrm{e}$, desses, um quarteirão, a esquina e a residência inicial para a realização das entrevistas 11. A partir daí, a entrevista ocorria a cada duas residências, totalizando 44 domicílios de cada setor. Ques- tionários pré-codificados e pré-testados em estudo piloto em 120 domicílios foram aplicados em todos os moradores com 20 anos ou mais de cada domicílio eleito.

Para o cálculo do tamanho da amostra, considerou-se: nível de 95\% de confiança, poder de $80 \%$, razão de prevalências de 2,0 para exposições, atingindo até $20 \%$ da população. Foram acrescentados $10 \%$ para possíveis perdas ou recusas e $15 \%$ para controle de fatores de confusão. Na falta de uma estimativa precisa para minimizar o efeito do delineamento, que ocorre no processo amostral por conglomerados, a amostra foi ampliada 1,5 vez para realizar as análises ajustadas. Chegou-se, assim, ao número de 2.110 indivíduos. Como esta pesquisa é parte de uma investigação sobre diversos aspectos de saúde dessa população, chegou-se a uma amostra total de 3.934 adultos e, dentre esses, 596 idosos com 60 anos ou mais de idade, sendo essa a amostra deste estudo 12 .

A estruturação do banco de dados foi feita no programa Epinfo 6.02 (CDC, Atlanta, Estados Unidos) com dupla entrada para correção de inconsistências.

O estado nutricional foi definido pelo IMC [peso $(\mathrm{kg})$ / estatura $\left(\mathrm{m}^{2}\right)$ ], calculado a partir de dados referidos pelos entrevistados. As medidas de peso e estatura auto-referidos foram validadas por meio de um subestudo, e os valores de IMC foram corrigidos por meio de uma equação de regressão linear, descrita por Silveira et al. 12 . A classificação do estado nutricional a partir do IMC foi realizada de acordo com dois critérios; o da OMS 4 que utiliza os mesmos pontos de corte para adultos: baixo peso IMC $<18,5 \mathrm{~kg} / \mathrm{m}^{2}$, eutrofia IMC entre $18,5 \mathrm{~kg} / \mathrm{m}^{2}$ e $24,9 \mathrm{~kg} / \mathrm{m}^{2}$, sobrepeso IMC entre 25 e $29,9 \mathrm{~kg} / \mathrm{m}^{2}$ e obesidade IMC $\geq 30 \mathrm{~kg} / \mathrm{m}^{2}$; e a recomendação de Lipschitz et al. ${ }^{6}$ que considera as modificações na composição corporal próprias do envelhecimento, a saber: baixo peso IMC $<22 \mathrm{~kg} / \mathrm{m}^{2}$, eutrofia IMC entre 22 e $27 \mathrm{~kg} / \mathrm{m}^{2}$ e excesso de peso $\mathrm{IMC}>27 \mathrm{~kg} / \mathrm{m}^{2}$. Dessa forma, trabalhou-se com duas variáveis dependentes: $\mathrm{IMC}>27 \mathrm{~kg} / \mathrm{m}^{2}$ e IMC $\geq 30 \mathrm{~kg} / \mathrm{m}^{2}$. Nesta pesquisa, ambos os desfechos são mencionados como obesidade, seguindo a tendência de outros autores 9,13,14,15.

As variáveis independentes nessa análise foram agrupadas em dois blocos: (i) sócio-demográficas: sexo (masculino, feminino), idade (60$65,66-70,71-75,76-88$ anos), escolaridade $(<1$, 1-4, 5-8, 9-11 anos completos), renda per capita em quartis (quartil 1: mínimo a R\$ 144,99, quartil 2: $\mathrm{R} \$ 145,00$ a $\mathrm{R} \$ 300,99$, quartil 3: $\mathrm{R} \$ 301,00$ a R\$ $1.500,00$ e quartil $4:>\mathrm{R} \$ 1.500,00$ ), estado civil (solteiro ou separado, casado ou viúvo), cor da pele (branca, preta ou parda); (ii) do estilo de 
vida: tabagismo (não, sim, ex-fumante), uso de bebida alcoólica no último mês (não, sim), exercício físico no último mês (não, sim).

Utilizou-se o programa Stata 8.0 (Stata Corp., College Station, Estados Unidos) na análise estatística. Considerou-se o desenho amostral e procedeu-se correção do efeito de delineamento durante as análises. Na análise bivariada, utilizou-se teste do qui-quadrado de Pearson e de tendência linear para verificar a associação entre as variáveis independentes e os dois desfechos. Essas associações foram apresentadas por meio da razão de prevalência como medida de efeito com intervalo de $95 \%$ de confiança.

Todas as variáveis com significância menor que 0,20 na análise bivariada foram consideradas candidatas ao modelo multivariado. A regressão de Poisson múltipla foi realizada de acordo com modelo teórico hierarquizado, estruturado em dois blocos de variáveis, conforme descrito anteriormente. A partir de um modelo que incluiu todas as variáveis do mesmo nível, foram retiradas, uma a uma, as variáveis com valor de $\mathrm{p}$ maior que 0,05 , por meio do teste da razão de máxima verossimilhança. A razão de prevalência e o valor de p apresentados estão ajustados pelas variáveis do mesmo nível ou superiores. Para avaliar a qualidade do ajuste em cada nível e do modelo final de regressão, utilizou-se o teste Goodness-of-fit. Calculou-se ainda o coeficiente de determinação do modelo hierarquizado final.

O projeto de pesquisa foi aprovado pelo Comitê de Ética em Pesquisa da Universidade Federal de Pelotas.

\section{Resultados}

Foram avaliados 596 idosos acima de 60 anos. A prevalência de obesidade (IMC $\geq 30 \mathrm{~kg} / \mathrm{m}^{2}$ ) foi de 25,3\% (IC95\%: 21,89-29,02), sendo 30,8\% em mulheres e $17,4 \%$ em homens e de $48,0 \%$ (IC95\%: $44,57-52,75)$ segundo o ponto de corte de IMC > $27 \mathrm{~kg} / \mathrm{m}^{2}$, sendo $53,4 \%$ e $40,1 \%$ em mulheres e homens, respectivamente (Figura 1 e 2).

Observou-se predomínio do sexo feminino $(59,4 \%)$ e da faixa etária entre 60 e 65 anos $(42,8 \%)$. A renda per capita média foi de $\mathrm{R} \$$ 495,35, enquanto, aproximadamente, um terço

Figura 1

Estado nutricional de homens e mulheres idosos segundo a classificação da Organização Mundial da Saúde. Pelotas, Rio Grande do Sul, Brasil, 2000 $(\mathrm{N}=596)$.

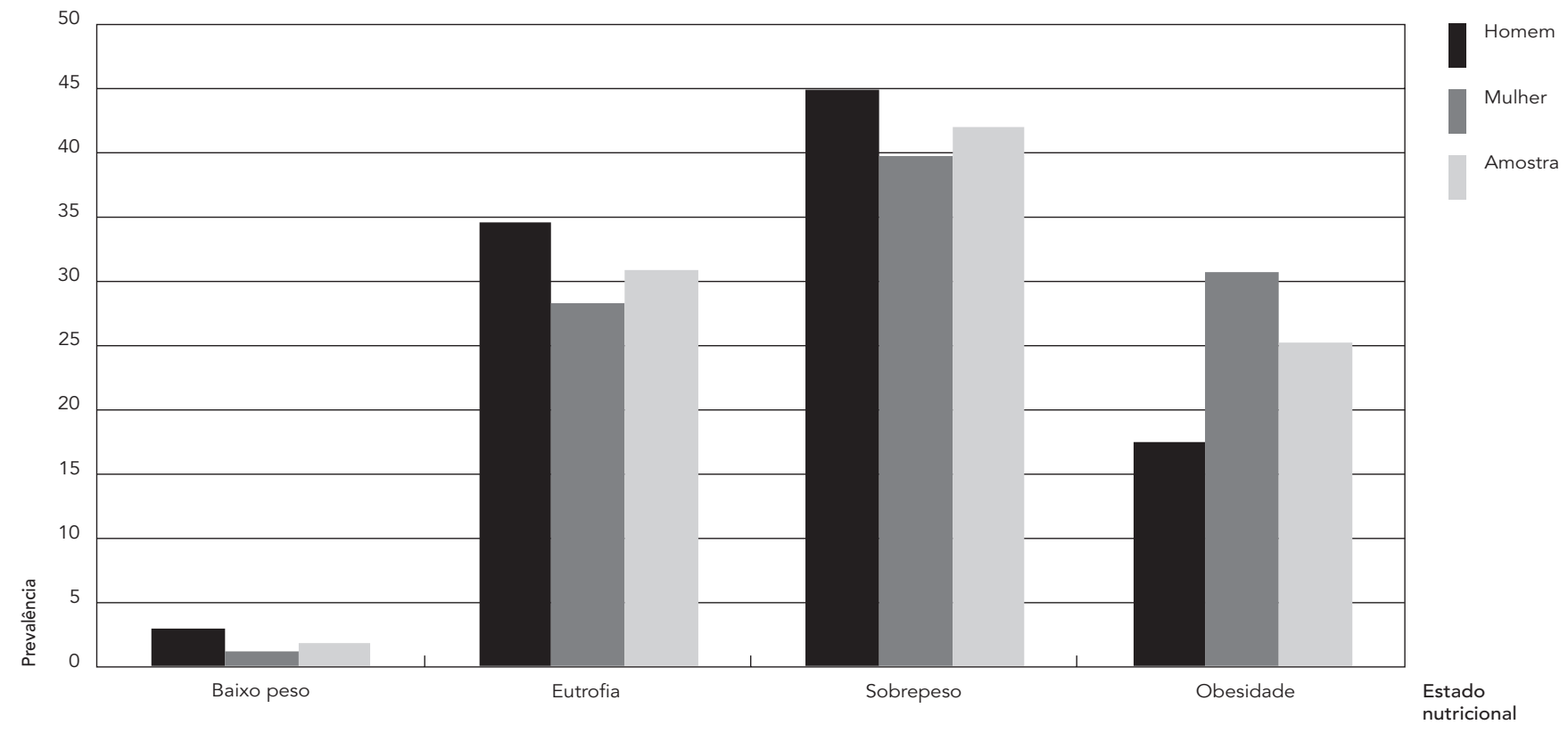

Nota: baixo peso (IMC < 18,5kg/m2); eutrofia (IMC: 18,5-24,9kg/m²); sobrepeso (IMC: 25-29,9kg/m2); obesidade (IMC $\left.\geq 30 \mathrm{~kg} / \mathrm{m}^{2}\right)$. 
Estado nutricional de homens e mulheres idosos segundo a classificação de Lipschitz 6. Pelotas, Rio Grande do Sul, Brasil, $2000(N=596)$.

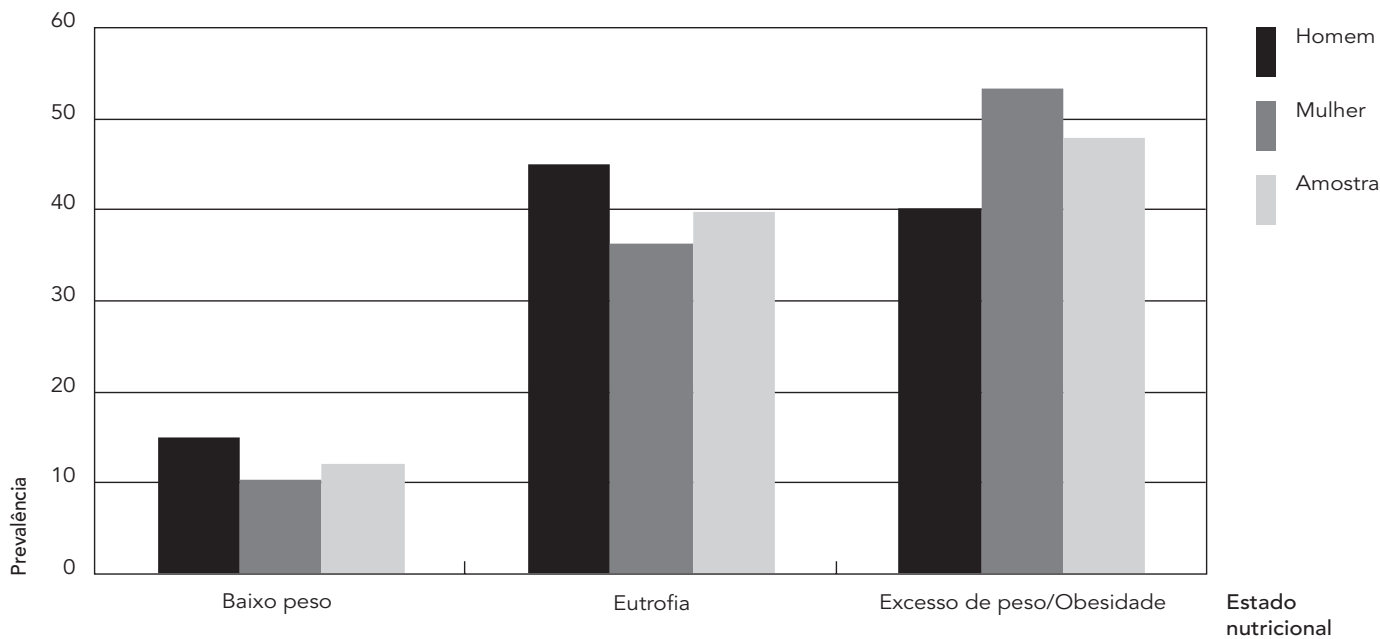

Nota: baixo peso (IMC: < 22kg/m²); eutrofia (IMC: $\left.22-27 \mathrm{~kg} / \mathrm{m}^{2}\right)$; excesso de peso/obesidade $\left(\mathrm{IMC}>27 \mathrm{~kg} / \mathrm{m}^{2}\right)$.

dos entrevistados apresentaram entre 1 e 4 anos de estudo. O uso de bebida alcoólica no último mês foi relatado por $32,3 \%$ dos participantes, a maioria (50,3\%) referiu não ser fumante, e 71,4\% não praticavam exercício físico na época do estudo (Tabela 1).

Independente do desfecho analisado, foram observadas maiores prevalências de obesidade no sexo feminino, nas categorias de mais baixo quartil de renda per capita, menos de um ano de escolaridade, cor da pele branca, solteiro ou separado e não fumante. Com relação à faixa etária, observou-se associação negativa, ou seja, quanto maior a idade menor a prevalência de obesidade, notadamente a partir de 75 anos. As maiores prevalências de obesidade foram observadas entre 66 e 70 anos (IMC $\geq 30 \mathrm{~kg} / \mathrm{m}^{2}$ ) e 60 a 65 anos para IMC $>27 \mathrm{~kg} / \mathrm{m}^{2}$. Idosos sedentários apresentam maior prevalência de obesidade apenas para IMC $\geq 30 \mathrm{~kg} / \mathrm{m}^{2}$ (Tabela 1 ).

As variáveis incluídas na regressão de Poisson, considerando os dois pontos de corte estudados, foram: sexo, idade, escolaridade e tabagismo. Apenas para IMC $\geq 30 \mathrm{~kg} / \mathrm{m}^{2}$, incluiu-se estado civil e exercício físico (Tabela 1).

Para a variável dependente IMC $>27 \mathrm{~kg} / \mathrm{m}^{2}$, observou-se, após análise hierarquizada em dois níveis, associação com: sexo feminino, idade, ex-fumante e não fumante. Observou-se ainda que, conforme aumenta a idade, diminui a razão de prevalência do desfecho comparado à faixa etária de 76 anos ou mais (Tabela 2).

A prevalência de obesidade (IMC $\geq 30 \mathrm{~kg} / \mathrm{m}^{2}$ ) foi maior entre as mulheres e, na análise ajustada, houve um pequeno aumento no efeito, sugerindo que a idade confunde negativamente essa associação. No segundo nível, ajustando para sexo e idade, não praticar exercício físico permaneceu associado à obesidade (Tabela 3).

Comparando os dois modelos multivariados, observaram-se algumas diferenças: idade até 75 anos, fumar ou ser ex-fumante mantiveram-se associados à obesidade no ponto de corte IMC $>27 \mathrm{~kg} / \mathrm{m}^{2}$, e não praticar exercício físico manteve-se associado somente com IMC $\geq 30 \mathrm{~kg} / \mathrm{m}^{2}$. Ao se observar as razões de prevalências ajustadas do IMC $\geq 30 \mathrm{~kg} / \mathrm{m}^{2}$ e do IMC $>27 \mathrm{~kg} / \mathrm{m}^{2}$, verifica-se uma redução na força da associação para sexo feminino, o que está de acordo quando se usa um ponto de corte mais sensível (Tabelas 2 e 3).

\section{Discussão}

O perfil nutricional dos idosos deste estudo foi caracterizado pela alta prevalência de obesidade, principalmente no sexo feminino e entre 60 e 75 anos. Este resultado é preocupante, principalmente pela associação da obesidade com 
Distribuição da amostra e prevalência de obesidade em idosos segundo IMC $>27 \mathrm{~kg} / \mathrm{m}^{2}$ e IMC $\geq 30 \mathrm{~kg} / \mathrm{m}^{2}$ pelas variáveis estudadas. Pelotas, Rio Grande do Sul, Brasil, 2000 ( $N=596$ )

\begin{tabular}{|c|c|c|c|c|c|}
\hline \multirow[t]{2}{*}{ Variáveis } & \multirow{2}{*}{$\begin{array}{c}\text { Distribuição da amostra } \\
\qquad n(\%)\end{array}$} & \multicolumn{2}{|c|}{$\mathrm{IMC}>27 \mathrm{~kg} / \mathrm{m}^{2}$} & \multicolumn{2}{|c|}{$\mathrm{IMC} \geq 30 \mathrm{~kg} / \mathrm{m}^{2}$} \\
\hline & & $\begin{array}{c}\text { Prevalência } \\
\text { n (\%) }\end{array}$ & Valor de $p$ * & $\begin{array}{c}\text { Prevalência } \\
\text { n (\%) }\end{array}$ & Valor de $p$ * \\
\hline Sexo & & & 0,001 & & 0,001 \\
\hline Masculino & $242(40,6)$ & $99(40,9)$ & & $42(17,4)$ & \\
\hline Feminino & $354(59,4)$ & $191(53,9)$ & & $109(30,8)$ & \\
\hline Idade (anos) & & & $<0,001 \star \star \star$ & & $0,040 * *$ \\
\hline $60-65$ & $255(42,8)$ & $141(55,3)$ & & $70(27,4)$ & \\
\hline $66-70$ & $120(20,1)$ & $61(50,8)$ & & $34(28,3)$ & \\
\hline $71-75$ & $120(20,1)$ & $60(50,0)$ & & $32(26,7)$ & \\
\hline $76-88$ & $101(17,0)$ & $28(27,7)$ & & $15(14,8)$ & \\
\hline Renda per capita (quartis) *** & & & $0,600 * \star$ & & 0,370 ** \\
\hline 1으 & $133(23,0)$ & $40(51,9)$ & & $41(30,8)$ & \\
\hline 2oㅇ & $142(24,6)$ & $75(47,8)$ & & $35(24,6)$ & \\
\hline 3으 & $162(28,1)$ & $84(50,6)$ & & $38(23,5)$ & \\
\hline 4으 & $140(24,3)$ & $83(46,9)$ & & $34(24,3)$ & \\
\hline Escolaridade (anos) & & & 0,080 ** & & $0,080 * *$ \\
\hline$<1$ & $115(19,4)$ & $64(55,6)$ & & $36(31,3)$ & \\
\hline $1-4$ & $201(33,8)$ & $99(49,2)$ & & $51(25,4)$ & \\
\hline $5-8$ & $160(26,9)$ & $73(45,6)$ & & $38(23,7)$ & \\
\hline$\geq 9$ & $118(19,9)$ & $53(44,9)$ & & $25(21,2)$ & \\
\hline Cor da pele & & & 0,602 & & 0,268 \\
\hline Branca & $505(84,7)$ & $248(49,1)$ & & $132(26,1)$ & \\
\hline Preta/Parda & $91(15,3)$ & $42(46,1)$ & & $19(20,9)$ & \\
\hline Estado civil & & & 0,415 & & 0,029 \\
\hline Casado ou viúvo & $375(62,9)$ & $178(47,5)$ & & $84(22,4)$ & \\
\hline Solteiro ou separado & $221(37,1)$ & $112(50,7)$ & & $67(30,3)$ & \\
\hline Uso de bebida alcoólica & & & 0,491 & & 0,390 \\
\hline Não & $403(67,7)$ & $193(47,9)$ & & $107(26,5)$ & \\
\hline $\operatorname{Sim}$ & $192(32,3)$ & $97(50,5)$ & & $42(22,9)$ & \\
\hline Tabagismo & & & 0,057 & & 0,199 \\
\hline Não & $299(50,3)$ & $158(52,8)$ & & $84(28,0)$ & \\
\hline Ex-fumante & $210(35,3)$ & $102(48,6)$ & & $52(24,8)$ & \\
\hline Fumante & $86(14,4)$ & $30(34,9)$ & & $15(17,4)$ & \\
\hline Exercício físico & & & 0,286 & & 0,043 \\
\hline Não & $425(71,4)$ & $202(47,5)$ & & $115(27,0)$ & \\
\hline Sim & $170(28,6)$ & $88(51,7)$ & & $36(21,2)$ & \\
\hline
\end{tabular}

* Corrigido para o desenho amostral;

** Qui-quadrado de tendência;

*** Dados ausentes para 19 indivíduos.

várias doenças crônicas não transmissíveis, que acarreta aumento da morbi-mortalidade e impacto sobre o sistema de saúde, além de diminuir a qualidade de vida dos idosos.

A obesidade é um problema de saúde em ascensão, sendo comum entre mulheres e populações urbanas 2,7,16. A maior prevalência de obesidade em mulheres idosas aqui observada é consistente com os resultados de outras pesquisas. No Brasil, resultados da PNSN e da POF 2002-2003, para indivíduos de 60 anos ou mais, revelaram maior prevalência nas mulheres quando comparadas aos homens, sendo que, nas regiões Sul e Sudeste, a obesidade se destaca do restante do país por atingir prevalências mais elevadas 2,7. Estudo multicêntrico em idosos brasileiros, acompanhados em ambulatórios, identificou $36 \%$ das mulheres com 
Tabela 2

Razão de prevalência (RP) bruta e ajustada hierarquicamente para prevalência de obesidade em idosos segundo IMC > $27 \mathrm{~kg} / \mathrm{m}^{2}$. Pelotas, Rio Grande do Sul, Brasil, 2000.

\begin{tabular}{|c|c|c|c|c|}
\hline Variáveis & RP bruta (IC95\%) * & Valor de $p *, \star \star$ & RP ajustada (IC95\%) * & Valor de $p *, \star \star$ \\
\hline \multicolumn{5}{|l|}{ 1으 Nível } \\
\hline Sexo & & 0,001 & & $<0,001$ \\
\hline Masculino & 1,00 & & 1,00 & \\
\hline Feminino & $1,32(1,12-1,56)$ & & $1,34(1,14-1,58)$ & \\
\hline Idade (anos) & & $<0,001 * \star \star$ & & $<0,001$ \\
\hline $60-65$ & $1,99(1,49-2,66)$ & & $2,02(1,52-2,68)$ & \\
\hline $66-70$ & $1,83(1,34-2,51)$ & & $1,87(1,38-2,54)$ & \\
\hline $71-75$ & $1,80(1,30-2,50)$ & & $1,84(1,34-2,54)$ & \\
\hline$\geq 76$ & 1,00 & & 1,00 & \\
\hline \multicolumn{5}{|l|}{ 2o Nível } \\
\hline Tabagismo & & 0,057 & & 0,036 \\
\hline Não & $1,51(1,08-2,13)$ & & $1,48(1,05-2,08)$ & \\
\hline Ex-fumante & $1,39(0,99-1,94)$ & & $1,52(1,01-2,10)$ & \\
\hline Fumante & 1,00 & & 1,00 & \\
\hline
\end{tabular}

Todos os valores ajustados pelas variáveis do mesmo nível e acima.

* Corrigido para o desenho amostral;

** Teste de Wald;

*** Qui-quadrado de tendência.

Tabela 3

Razão de prevalência (RP) bruta e ajustada hierarquicamente para prevalência de obesidade em idosos segundo IMC $\geq$ $30 \mathrm{~kg} / \mathrm{m}^{2}$. Pelotas, Rio Grande do Sul, Brasil, 2000.

\begin{tabular}{|c|c|c|c|c|}
\hline Variáveis & RP bruta (IC95\%) * & Valor de $p *, \star \star$ & RP ajustada (IC95\%)* & Valor de $p^{*, * *}$ \\
\hline \multicolumn{5}{|l|}{ 1으 Nível } \\
\hline Sexo & & 0,001 & & 0,001 \\
\hline Masculino & 1,00 & & 1,00 & \\
\hline Feminino & $1,77(1,25-2,52)$ & & $1,81(1,2-2,55)$ & \\
\hline Idade (anos) & & $0,049 * \star \star$ & & 0,027 \\
\hline $60-65$ & $1,85(1,09-3,13)$ & & $1,89(1,12-3,17)$ & \\
\hline $66-70$ & $1,91(1,00-3,62)$ & & $1,99(1,05-3,76)$ & \\
\hline $71-75$ & $1,79(1,03-3,11)$ & & $1,88(1,08-3,26)$ & \\
\hline$\geq 76$ & 1,00 & & 1,00 & \\
\hline \multicolumn{5}{|l|}{ 2o Nível } \\
\hline Exercício físico & & 0,043 & & 0,042 \\
\hline Não & $1,28(1,01-1,62)$ & & $1,27(1,01-1,60)$ & \\
\hline Sim & 1,00 & & 1,00 & \\
\hline
\end{tabular}

Nota: Todos os valores ajustados pelas variáveis do mesmo nível e acima.

* Corrigido para o desenho amostral;

** Teste de Wald;

*** Qui-quadrado de tendência. 
IMC $\geq 27,3 \mathrm{~kg} / \mathrm{m}^{2}$ e $22 \%$ dos homens com IMC $\geq 27,8 \mathrm{~kg} / \mathrm{m}^{2} 17$. Uma possível explicação para maior prevalência de obesidade nas mulheres pode ser o maior acúmulo de gordura visceral e maior expectativa de vida 18,19 . Durante o processo de envelhecimento, ocorre redistribuição progressiva da gordura com diminuição do panículo adiposo subcutâneo dos membros e acúmulo intra-abdominal. As mulheres acumulam mais gordura subcutânea que os homens e a perdem em idades mais tardias 18 .

Os idosos de idade mais avançada apresentaram menor prevalência de obesidade, ao contrário do que ocorre em adultos. Essa mesma tendência foi observada na PNSN, POF e em outras pesquisas 2,5,7,16,20. O envelhecimento promove mudanças importantes na massa muscular e no padrão de distribuição de gordura corporal 5,21. O peso diminui com a idade após atingir um platô; nos homens, ao redor de 65 anos; nas mulheres, cerca de dez anos mais tarde 7,19,21. No presente estudo, observou-se decréscimo da freqüência de obesos de maneira homogênea e progressiva nas diversas faixas etárias, analisando o ponto de corte de IMC $>27 \mathrm{~kg} / \mathrm{m}^{2}$, porém o mesmo não ocorreu para IMC $\geq 30 \mathrm{~kg} / \mathrm{m}^{2}$. Isso possivelmente se deve à variação amostral e ao reduzido número de idosos com 76 anos ou mais de idade com IMC $\geq 30 \mathrm{~kg} / \mathrm{m}^{2}(\mathrm{n}=15)$.

A redução da obesidade com o avançar da idade também poderia ser explicada pelo efeito do viés de sobrevivência, devido ao impacto negativo da obesidade e das patologias a ela associadas, contribuindo para a maior mortalidade observada em idosos obesos antes dos 80 anos 22. The Rotterdam Study 23 descreve que a relação entre IMC e mortalidade parece ser menos pronunciada em idosos do que entre adultos jovens, uma provável explicação pode ser a sobrevivência seletiva dos idosos. Existem controvérsias quanto ao significado da obesidade em idosos longevos em que a desnutrição parece atuar de forma mais significativa sobre a mortalidade do que a obesidade 23 .

Nesta pesquisa, observou-se, após análise ajustada, que ex-fumantes apresentaram maiores prevalências de obesidade (IMC $>27 \mathrm{~kg} / \mathrm{m}^{2}$ ) quando comparados ao grupo dos fumantes. Maior prevalência de obesidade entre ex-fumantes também foi observado em São Paulo, porém as análises foram feitas para população acima de 18 anos, sem estratificação para idosos 20 .

Entre idosos sedentários, a prevalência de obesidade (IMC $\geq 30 \mathrm{~kg} / \mathrm{m}^{2}$ ) foi maior quando comparada a dos praticantes de atividade física, permanecendo associada após análise ajustada, estando de acordo com estudo realizado por Barreto et al. 16, em que a obesidade (IMC >
$30 \mathrm{~kg} / \mathrm{m}^{2}$ ) associou-se inversamente com a atividade física.

Uma possível limitação, no presente estudo, consiste na utilização das medidas de peso e estatura referidos para o cálculo do IMC e definição do estado nutricional. Cabe destacar que foi realizado por Silveira et al. 12 um estudo de validação dessas medidas para essa população, desenvolvendo-se uma equação de regressão linear para correção do IMC, quando necessário. Todos esses cuidados foram tomados na presente análise. Outra limitação, inerente aos estudos transversais, refere-se à possibilidade de viés de causalidade reversa. Esse viés deve ser considerado especialmente na interpretação da associação entre prática de exercício físico e obesidade.

É importante ampliar o debate e o desenvolvimento de pesquisas sobre a avaliação antropométrica e nutricional de idosos com aplicabilidade clínica, epidemiológica e no âmbito da rede básica de saúde. Um ponto de corte único facilitaria comparações de resultados de pesquisas nacionais e internacionais, mas, para isso, as pesquisas precisam avançar avaliando: adiposidade, composição corporal e distribuição de gordura, risco e impacto na morbi-mortalidade em idosos de diferentes faixas etárias. Para se chegar a um consenso sobre os pontos de corte de IMC para avaliar o estado nutricional de idosos, além de avaliar os aspectos citados, faz-se necessário o desenvolvimento de pesquisas que considerem idosos brasileiros. A OMS propôs, em 2002, que cada país tome suas decisões sobre as definições de risco populacional, considerando diferenças étnicas e características próprias 24.

Avaliando grupos de idosos de Florianópolis, Santa Catarina, Brasil, com relação aos pontos de corte de IMC 25,27 e $30 \mathrm{~kg} / \mathrm{m}^{2}$ e tendo como padrão-ouro o percentual de gordura corporal avaliado pela absorptiometria de raio$\mathrm{X}$ de dupla energia (DEXA), os maiores valores de concordância encontrados foram para IMC $27 \mathrm{~kg} / \mathrm{m}^{2}$ no sexo masculino. Nessa mesma pesquisa, o IMC $\geq 30 \mathrm{~kg} / \mathrm{m}^{2}$ apresentou os menores valores de sensibilidade para ambos os sexos, enquanto, para o IMC $27 \mathrm{~kg} / \mathrm{m}^{2}$, obteve-se sensibilidade e especificidade no sexo masculino de $73,7 \%$ e $72,5 \%$ e, no sexo feminino, $56,1 \%$ e $100 \%$, respectivamente 15 . O ponto de corte de IMC, $27 \mathrm{~kg} / \mathrm{m}^{2}$, de maior concordância com percentual de gordura corporal no estudo de Florianópolis, está de acordo com Lipschitz et al. 6, sendo utilizado no NSI e também no NHANES III, e baseia-se em maior mortalidade dos idosos acima desse ponto 6,9,10,15.

Em diversos países, sabe-se que o diagnóstico de obesidade não é o mesmo quando se compara percentual de gordura corporal e IMC. 
Parece ser equivocado o pressuposto que o IMC mede com a mesma capacidade a adiposidade em todas as faixas etárias, notadamente no envelhecimento 5,25,26. Adicionalmente, a centralização da gordura corporal parece predizer melhor as complicações em idosos 23 . Mudanças relacionadas com o acúmulo da gordura visceral ou subcutânea, associadas ao processo de envelhecimento, podem ser afetadas, tanto pela quantidade inicial de tecido adiposo, como pelo aumento da massa corporal 21,26,27.

Considerando as modificações de composição corporal no processo de envelhecimento e as evidências disponíveis até o momento, não parece adequado utilizar o mesmo ponto de corte de IMC de populações adultas para classificar obesidade em idosos. Dessa forma, até que novas definições e avanços em pesquisas surjam, sugere-se, sob a ótica da saúde pública, a adoção de um ponto de corte de obesidade mais sensível, ou seja, IMC > 27kg/m² para a população idosa brasileira.

Estudos com um número maior de idosos, nas faixas etárias mais avançadas e que associem outros métodos antropométricos na avaliação da obesidade, além do IMC, serão de grande importância técnica para esse campo de investigações. No futuro, talvez o IMC e mesmo os pontos de corte até então recomendados não sejam os mais apropriados para a avaliação nutricional de idosos.

Embora o baixo peso seja um problema relacionado com o estado nutricional de idosos, este estudo revelou que a obesidade é muito mais prevalente. Assim, faz-se necessário estabelecer práticas de monitoramento do estado nutricional, direcionar intervenções cada vez mais adequadas, além de prevenção e controle da obesidade em programas voltados para a promoção da saúde e qualidade de vida dos idosos.

\section{Resumo}

Estimar a prevalência e fatores associados à obesidade em idosos segundo dois pontos de corte do índice de massa corporal (IMC). Trata-se de estudo transversal de base populacional em amostra de idosos residentes em Pelotas, Rio Grande do Sul, Brasil. Avaliou-se o estado nutricional, pelo IMC, em 596 idosos, a partir de peso e estatura, referidos e validados. Utilizou-se regressão de Poisson múltipla com análise hierarquizada, com duas variáveis dependentes para definição da obesidade: $I M C>27 \mathrm{~kg} / \mathrm{m}^{2}$ e IMC $\geq 30 \mathrm{~kg} / \mathrm{m}^{2}$, proposta de Lipschitz e da Organização Mundial da Saúde, respectivamente. A prevalência de obesidade foi $48,7 \%$ (IC95\%: 44,6-52,7) para IMC > 27kg/m $\mathrm{m}^{2}$ e de $25,3 \%$ (IC95\%: 21,9-29,0) para IMC $\geq 30 \mathrm{~kg} / \mathrm{m}^{2}$. Observou-se, nos dois modelos de regressão múltipla, associação da obesidade com sexo feminino e idade. Para o IMC > $27 \mathrm{~kg} / \mathrm{m}^{2}$, observou-se associação com idade e tabagismo e, para o desfecho $I M C \geq 30 \mathrm{~kg} / \mathrm{m}^{2}$, com não prática de exercício físico. A prevalência de obesidade nos idosos foi elevada. Sugere-se, sob a ótica da saúde pública, a adoção de um ponto de corte de obesidade mais sensível, ou seja, IMC > 27kg/m² para a população idosa brasileira.

Idoso; Obesidade; Índice de Massa Corporal; Avaliação Nutricional; Prevalência

\section{Colaboradores}

E. A. Silveira participou de todas as etapas da pesquisa, desde o planejamento até a revisão final do artigo. G. Kac participou da interpretação dos dados, elaboração e revisão da versão final. L. S. Barbosa colaborou na análise estatística e contribuiu na elaboração do artigo.

\section{Agradecimentos}

Erika Aparecida Silveira recebeu bolsa de mestrado da Coordenação de Aperfeiçoamento de Pessoal de nível Superior (CAPES, 1999-2000). O projeto de pesquisa original foi parcialmente financiado pelo Programa de Pós-graduação em Epidemiologia da Universidade Federal de Pelotas. Gilberto Kac é pesquisador com bolsa de produtividade do Conselho Nacional de Desenvolvimento Científico e Tecnológico (CNPq). Agradecimentos especiais a Denise P. Gigante do Programa de Pósgraduação em Epidemiologia da Universidade Federal de Pelotas pela sua colaboração e contribuições. 


\section{Referências}

1. Caldwell JC. Population health in transition. Bull World Health Organ 2001; 79:159-60.

2. Coordenação de Índices de Preços, Instituto Brasileiro de Geografia e Estatística. Pesquisa de orçamentos familiares 2002-2003: primeiros resultados: Brasil e grandes regiões. Rio de Janeiro: Instituto Brasileiro de Geografia e Estatística; 2004.

3. Visscher TL, Seidell JC, Menotti A, Blackburn H, Nissinen A, Feskens EJ, et al. Underweight and overweight in relation to mortality among men aged 40-59 and 50-69 years: the seven countries study. Am J Epidemiol 2000; 151:660-6.

4. World Health Organization. Obesity: preventing and managing the global epidemic. Geneva: World Health Organization; 1998. (Technical Report Series, 894).

5. Santos DM, Sichieri R. Índice de massa corporal e indicadores antropométricos de adiposidade em idosos. Rev Saúde Pública 2005; 39:163-8.

6. Lipschitz DA. Screening for nutritional status in the elderly. Prim Care 1994; 21:55-67.

7. Tavares EL, Anjos LA. Perfil antropométrico da população idosa brasileira. Resultados da Pesquisa Nacional sobre Saúde e Nutrição. Cad Saúde Pública 1999; 15:759-68.

8. Cervi A, Franceschini SCC, Priori SE. Análise crítica do uso do índice de massa corporal para idosos. Rev Nutr 2005; 18:765-75.

9. National Center for Health Statistic, Centers for Disease Control and Prevention. National Health and Nutrition Examination Survey (NHANES III). Atlanta: National Heart, Lung and Blood Institute; 1991.

10. Nutrition screening manual for professionals caring for older Americans. Washington DC: Nutrition Screening Initiative; 1991.

11. Instituto Brasileiro de Geografia e Estatística. Resultados do universo relativo às características da população e dos domicílios - Rio Grande do Sul. Rio de Janeiro: Instituto Brasileiro de Geografia e Estatística; 1997.

12. Silveira EA, Araújo CL, Gigante DP, Barros AJD, Lima MS. Validação de peso e altura referidos para o diagnóstico do estado nutricional em uma população de adultos no Sul do Brasil. Cad Saúde Pública 2005; 21:235-45.

13. Alencar YMG, Carcalho Fo ET, Paschoal SMP, Curiati JAE, Ping WC, Litvoc J. Risk factors for atherosclerosis in an elderly out patient population in the city of São Paulo. Arq Bras Cardiol 2000; 74:189-96.

14. Da Cruz IBM, Almeida MSC, Schwanke CHA, Moriguchi EH. Prevalência de obesidade em idosos longevos e sua associação com fatores de risco de morbidades cardiovasculares. Rev Assoc Med Bras (1992) 2004; 50:172-7.

15. Cordeiro BA. Sensibilidade e especificidade do índice de massa corporal (IMC) no diagnóstico de obesidade em idosos: comparação com a absortometria por raio-X de dupla energia (DEXA) e proposta de novos pontos de corte [Dissertação de Mestrado]. Florianópolis: Programa de Pós-graduação em Nutrição, Universidade Federal de Santa Catarina; 2006.
16. Barreto SM, Passos VMA, Lima-Costa MFF. Obesity and underweight among Brazilian elderly. The Bambuí Health and Aging Study. Cad Saúde Pública 2003; 19:605-12.

17. Taddei CFG, Ramos LR, Moraes JC, Wajngarten M, Libberman A, Santos SC, et al. Estudo multicêntrico de idosos atendidos em ambulatórios de cardiologia e geriatria de instituições brasileiras. Arq Bras Cardiol 1997; 69:327-33.

18. World Health Organization. Physical status: the use and interpretation of anthropometry. Geneva: World Health Organization; 1995. (Technical Report Series, 854).

19. Silveira EA, Lopes ACS, Caialfa WT. Avaliação do estado nutricional de idosos. In: Kac G, Sichieri R, Gigante DP, organizadores. Epidemiologia nutricional. Rio de Janeiro: Editora Atheneu; 2007. p. 105-25.

20. César CLG, Carandina L, Alves MCGP, Barros MBA, Goldbaum M. Saúde e condição de vida em São Paulo: inquérito multicêntrico de saúde no Estado de São Paulo-ISA-SP. São Paulo: Faculdade de Saúde Pública, Universidade de São Paulo; 2005.

21. Perissinotto E, Pisent C, Sergi G, Grigoletto F, Enzi G. Anthropometric measurements in the elderly: age and gender differences. Br J Nutr 2002; 87: 177-86.

22. Cabrera MAS, Jacob Filho W. Obesidade em idosos; prevalência, distribuição e associação com hábitos e co-morbidades. Arq Bras Endocrinol Metabol 2001; 45:494-501.

23. Visscher TLS, Seidell JC, Molarius A, van der Kuip D, Hofman A, Wittema JCM. A comparasion of body mass index, waist-hip ratio and waist circuference as predictors of all-cause mortality among the elderly: the Rotterdam study. Int J Obes Relat Metab Disor 2001; 25:1730-5.

24. World Health Organization. Active ageing: a policy framework. A contribution of the World Health Organization to the Second United Nations World Assembly on Ageing. Madrid: World Health Organization; 2002.

25. Movsesyan L, Laszlo B, Philip JL, Christiansen C, Svendsen OL. Variations in percentage of body fat within different BMI groups in young, middle-aged and old women. Clin Physiol Funct Imaging 2003; 23:130-3.

26. Bedogni G, Pietrobelli A, Heymsfield SB, Borghi A, Manzieri AM, Morini P, et al. Is body mass index a measure of adiposity in elderly women? Obes Res 2001; 9:17-20.

27. Pounder D, Carson D, Davison M, Orihara Y. Evaluation of indices of obesity in men: descriptive study. BMJ 1998; 316:1428-9.

Recebido em 13/Jun/2008

Versão final reapresentada em 13/Nov/2008

Aprovado em 19/Fev/2009 